\title{
CANONICAL SURFACES OF HIGHER DEGREE
}

\author{
FABRIZIO CATANESE
}

\section{Dedicated to Philippe Ellia on the occasion of his 60th birthday}

\begin{abstract}
We consider a family of surfaces of general type $S$ with $K_{S}$ ample, having $K_{S}^{2}=24, p_{g}(S)=6, q(S)=0$. We prove that for these surfaces the canonical system is base point free and yields an embedding $\Phi_{1}: S \rightarrow \mathbb{P}^{5}$.

This result answers a question posed by G. and M. Kapustka Kap-Kap15.

We discuss some related open problems, concerning also the case $p_{g}(S)=5$, where one requires the canonical map to be birational onto its image.
\end{abstract}

\section{Contents}

Introduction

1. The construction of the family of surfaces

2. Proof that the canonical map is an embedding

3. The canonical ring as a module over the symmetric algebra $\mathcal{A}:=\operatorname{Sym}\left(\mathcal{R}_{1}\right)$

References

\section{INTRODUCTION}

Among the many questions that one may ask about surfaces of general type the following one has not yet been sufficiently considered.

Question 0.1. Let $S$ be a smooth surface with ample canonical divisor $K_{S}$, assume that $p_{g}(S)=6$ and that the canonical map $\phi_{1}: S \rightarrow \mathbb{P}^{5}$ is a biregular embedding.

Which values of $K_{S}^{2}$ can occur, in particular which is the maximal value that $K_{S}^{2}$ can reach?

Recall the Castelnuovo inequality, holding if $\phi_{1}$ is birational (onto its image):

$$
K_{S}^{2} \geq 3 p_{g}(S)-7
$$

Date: September 24, 2018

AMS Classification: 14J29, 14J10, 14M07.

Key words: Canonical surfaces, canonical ring.

The present work took place in the realm of the ERC Advanced grant n. 340258, 'TADMICAMT'. 
and the Bogomolov-Miyaoka-Yau inequality

$$
K_{S}^{2} \leq 9 \chi(S)=9+9 p_{g}(S)-9 q(S) .
$$

By virtue of these inequalities, under the assumptions of question 0.1 one must have:

$$
11 \leq K_{S}^{2} \leq 63
$$

In the article Cat97] methods of homological algebra were used to construct such surfaces with low degree $11 \leq K_{S}^{2} \leq 17$, and also to attempt a classification of them. Recently, M. and G. Kapustka constructed in Kap-Kap15 such canonical surfaces of degree $K_{S}^{2}=18$, using the method of bilinkage. In a preliminary version of the article they even ventured to ask whether the answer to question 0.1 would be $K_{S}^{2} \leq 18$.

Our main result consists in exhibiting such canonically embedded surfaces having degree $K_{S}^{2}=24$, and with $q(S)=0$. The family of surfaces was indeed listed in the article [Cat99], dedicated to applications of the technique of bidouble covers; but it was not a priori clear that their canonical system would be an embedding (this was proven in [Cat84 for bidouble covers satisfying much stronger conditions).

Theorem 0.1. Assume that $S$ is a bidouble cover of the quadric $Q=\mathbb{P}^{1} \times \mathbb{P}^{1}$ branched on three curves $D_{1}, D_{2}, D_{3}$ of respective bidegrees $(2,3),(2,3)(4,1)$, which are smooth and intersect transversally. Assume moreover that the 12 intersection points $D_{1} \cap D_{2}$ have pairwise different images via the second projection $p_{v}: Q \rightarrow \mathbb{P}^{1}$ (i.e., they have different coordinates $\left.\left(v_{0}: v_{1}\right)\right)$.

Then $S$ is a simply connected 1 surface with $K_{S}^{2}=24, p_{g}(S)=$ $6, q(S)=0$, whose canonical map $\phi_{1}: S \rightarrow \mathbb{P}^{5}$ is a biregular embedding. These surfaces form a (non empty) irreducible algebraic subset of dimension 25 of the moduli space.

We plan to try to describe the equations of the above surfaces $S \subset \mathbb{P}^{5}$; since, by a theorem of Walter Wal96, each $S$ is the Pfaffian locus of a twisted antisymmetric map $\alpha: \mathcal{E}(-t) \rightarrow \mathcal{E}$ of a vector bundle $\mathcal{E}$ on $\mathbb{P}^{5}$, a natural question is to describe the bundle $\mathcal{E}$.

Concerning question 0.1, we should point out that it is often easier to construct algebraic varieties as parametric images rather than as zero sets of ideals. Note that surfaces with $p_{g}(S)=6, q(S)=2, K_{S}^{2}=45$ (and with $K_{S}$ ample since they are ball quotients) were constructed in [BC08]. For these, however, the canonical system has base points.

It would be interesting to see whether there do exist canonically embedded surfaces with $K_{S}^{2}=56$ which are regular surfaces isogenous to a product (see [Cat00]).

\footnotetext{
${ }^{1}$ This follows from theorem 3.8 of Cat84.
} 
We finish this introduction pointing out that a similar question is wide open also for $p_{g}(S)=5$ (while for $p_{g}(S)=4$ some work has been done, see for instance [Cat99]).

Question 0.2. Let $S$ be a smooth minimal surface of general type, assume that $p_{g}(S)=5$ and that the canonical map $\phi_{1}: S \rightarrow \mathbb{P}^{4}$ is birational. What is the maximal value that $K_{S}^{2}$ can reach?

Again, Castelnuovo's inequalty gives $8 \leq K_{S}^{2}$, and Bogomolov-MiyaokaYau's inequality gives $K_{S}^{2} \leq 54$.

The known cases where $\phi_{1}$ is an embedding are just for $K_{S}^{2}=8,9$, where $S$ is a complete intersection of type $(4,2)$ or $(3,3)$.

Indeed these are the only cases by virtue of the following (folklore?) theorem which was stated and proven in Cat97, propositions 6.1 and 6.2 , corollary 6.3 .

Theorem 0.2. Assume that $S$ is the minimal model of a surface of general type with $p_{g}(S)=5$, and assume that the canonical map $\phi_{1}$ embeds $S$ in $\mathbb{P}^{4}$.

Then $S$ is a complete intersection with $\mathcal{O}_{S}\left(K_{S}\right)=\mathcal{O}_{S}(1)$, i.e., $S$ is a complete intersection in $\mathbb{P}^{4}$ of type $(2,4)$ or $(3,3)$.

Moreover, if $\phi_{1}$ is birational, and $K_{S}^{2}=8,9$, then $\phi_{1}$ yields an embedding of the canonical model of $X$ as a complete intersection in $\mathbb{P}^{4}$ of type $(2,4)$ or $(3,3)$.

Recall that the first main ingredient of proof is the well known Severi's double point formula ([Sev01, see [Cat79] for the proof of some transversality claims made by Severi).

Write the double point formula in the form stated in [Hart77], Appendix A, 4.1.3. It gives, once we set $d:=K_{S}^{2}$ :

$$
12 \chi(S)=(17-d) d .
$$

Since $\chi(S) \geq 1$, we get $8 \leq d \leq 16$, and viewing the double point formula as an equation among integers, we see that it is only solvable for $d=8,9 \Rightarrow \chi(S)=6 \Rightarrow q(S)=0$, or for $d=12, \Rightarrow \chi(S)=5 \Rightarrow$ $q(S)=1$.

One sees that the last case cannot occur, since the Albanese map of $S, \alpha: S \rightarrow A$, has as image an elliptic curve $A$; if one denotes by $g$ the genus of the Albanese fibres, the slope inequalities for fibred surfaces of Horikawa, or Xiao, or Konno ([Hor81], Xiao87], Kon93]), give $g=2$ : hence $\phi_{1}$ cannot be birational.

One could replace in theorem 0.2 the hypothesis that $\phi_{1}$ is an embedding of $S$ in $\mathbb{P}^{4}$ by the weaker condition that $\phi_{1}$ yields an embedding of the canonical model of $X$ via an extension of the Severi double formula to the case of surfaces with rational double points as singularities 2 .

\footnotetext{
${ }^{2}$ for which however we have not yet found a reference.
} 


\section{The CONSTRUCtion OF THE FAMily of SURFACES}

We consider the family of algebraic surfaces $S$, bidouble covers (Galois covers with group $\left.(\mathbb{Z} / 2)^{2}\right)$ of the quadric $Q:=\mathbb{P}^{1} \times \mathbb{P}^{1}$, described in the fourth line of page 106 of Cat99.

This means that we take three divisors

$$
D_{j}=\left\{\delta_{j}=0\right\}, \delta_{1}, \delta_{2} \in H^{0}\left(\mathcal{O}_{Q}(2,3)\right), \delta_{3} \in H^{0}\left(\mathcal{O}_{Q}(4,1)\right),
$$

we consider divisor classes $L_{1}=L_{2}, L_{3}$ with

$$
\mathcal{O}_{Q}\left(L_{1}\right)=\mathcal{O}_{Q}\left(L_{2}\right)=\mathcal{O}_{Q}(3,2), \mathcal{O}_{Q}\left(L_{3}\right)=\mathcal{O}_{Q}(2,3)
$$

and we define the surface $S$ as

$$
\operatorname{Spec}\left(\mathcal{O}_{Q} \oplus w_{1} \mathcal{O}_{Q}\left(-L_{1}\right) \oplus w_{2} \mathcal{O}_{Q}\left(-L_{2}\right) \oplus w_{3} \mathcal{O}_{Q}\left(-L_{3}\right)\right),
$$

where the ring structure is given by (here $\{i, j, k\}$ is any permutation of $\{1,2,3\})$

$$
w_{i}^{2}=\delta_{j} \delta_{k}, w_{i} w_{j}=w_{k} \delta_{k} .
$$

We refer to Cat84 and Cat99] for the basics on bidouble covers, which show that on the surface $S$ the sections $w_{i}$ can be written as a product of square roots of the sections $\delta_{1}, \delta_{2}, \delta_{3}$ :

$$
w_{i}=y_{j} y_{k}, y_{i}^{2}=\delta_{i} \text {. }
$$

As is in Cat99, page 102, define

$$
N:=2 K_{Q}+\sum_{1}^{3} D_{i}=2 K_{Q}+\sum_{1}^{3} L_{i},
$$

obtaining a description of the canonical ring of $S$, which is a smooth surface with ample canonical divisor if the three curves $D_{1}, D_{2}, D_{3}$ are smooth and intersect transversally. The canonical ring is defined as usual by:

$$
\mathcal{R}:=\oplus_{m=0}^{\infty} \mathcal{R}_{m}, \mathcal{R}_{m}:=H^{0}\left(\mathcal{O}_{S}\left(m K_{S}\right)\right),
$$

and we have (ibidem)

$$
\begin{aligned}
\mathcal{R}_{2 m+1}= & y_{1} y_{2} y_{3} H^{0}\left(\mathcal{O}_{Q}\left(K_{Q}+m N\right)\right) \oplus\left(\oplus_{i=1}^{3} y_{i} H^{0}\left(\mathcal{O}_{Q}\left(K_{Q}+m N+L_{i}\right)\right)\right) \\
& \mathcal{R}_{2 m}=H^{0}\left(\mathcal{O}_{Q}(m N)\right) \oplus\left(\oplus_{i=1}^{3} w_{i} H^{0}\left(\mathcal{O}_{Q}\left(m N-L_{i}\right)\right)\right) .
\end{aligned}
$$

In particular, since in this case $\mathcal{O}_{Q}(N)=\mathcal{O}_{Q}(4,3)$, $H^{0}\left(\mathcal{O}_{S}\left(K_{S}\right)\right)=\oplus_{i=1}^{3} y_{i} H^{0}\left(\mathcal{O}_{Q}\left(K_{Q}+L_{i}\right)\right)=<y_{1} u_{0}, y_{1} u_{1}, y_{2} u_{0}, y_{2} u_{1}, y_{3} v_{0}, y_{3} v_{1}>$, where $u_{0}, u_{1}$ is a basis of $H^{0}\left(\mathcal{O}_{Q}(1,0)\right), v_{0}, v_{1}$ is a basis of $H^{0}\left(\mathcal{O}_{Q}(0,1)\right)$.

In particular, $p_{g}(S)=6$; moreover, $q(S)=0$ since $H^{1}\left(\mathcal{O}_{Q}\right)=$ $H^{1}\left(\mathcal{O}_{Q}\left(-L_{i}\right)\right)=0, \forall i=1,2,3$, as follows easily from the Künneth formula.

Finally, if $\pi: S \rightarrow Q$ is the bidouble cover, then $2 K_{S}=\pi^{*}(N)$, hence $K_{S}$ is ample and $K_{S}^{2}=N^{2}=24$. 


\section{Proof that the CANONiCAL MaP is AN EMBEDding}

Theorem 2.1. Assume that the three branch curves $D_{1}, D_{2}, D_{3}$ are smooth and intersect transversally, and moreover that the 12 intersection points $D_{1} \cap D_{2}$ have pairwise different images via the second projection $p_{v}: Q \rightarrow \mathbb{P}^{1}$ (i.e., they have different coordinates $\left(v_{0}: v_{1}\right)$ ).

Then the canonical map $\phi_{1}: S \rightarrow \mathbb{P}^{5}, \phi_{1}(x)=$ $=\left(y_{1}(x) u_{0}(x): y_{1}(x) u_{1}(x): y_{2}(x) u_{0}(x): y_{2}(x) u_{1}(x): y_{3}(x) v_{0}(x): y_{3}(x) v_{1}(x)\right)$,

is a biregular embedding, i.e. we have an isomorphism

$$
\phi_{1}: S \rightarrow \Sigma:=\phi_{1}(S) .
$$

Proof. Let $R_{i} \subset S, R_{i}:=\left\{y_{i}=0\right\}$. The curve $R_{i}$ maps to $D_{i}$ with degree 2 , and $R_{1} \cap R_{2} \cap R_{3}=\emptyset$ since by our assumption $D_{1} \cap D_{2} \cap D_{3}=\emptyset$.

Claim 1: the canonical system is base-point free.

In fact, for each point $x$ there is $u_{i}, i \in\{0,1\}$, such that $u_{i}(x) \neq 0$, and similarly $v_{j}, j \in\{0,1\}$, such that $v_{j}(x) \neq 0$. Hence $x$ is a base point if $y_{1}=y_{2}=y_{3}=0$ at $x$, contradicting $R_{1} \cap R_{2} \cap R_{3}=\emptyset$.

Claim 2: $\phi_{1}$ is a local embedding.

2.1) At the points $x \in R_{h} \cap R_{k}$ the two sections $y_{h}, y_{k}$ yield local coordinates, hence our assertion.

2.2) For the points $x \in S \backslash R$, where $R=R_{1} \cup R_{2} \cup R_{3}$ is the ramification divisor, let us consider the rational map $F_{u}: \Sigma \rightarrow \mathbb{P}^{1}$, induced by the linear projections

$$
\begin{aligned}
& \left(x_{1}: x_{2}: x_{3}: x_{4}: x_{5}: x_{6}\right) \rightarrow\left(x_{1}: x_{2}\right) \\
& \left(x_{1}: x_{2}: x_{3}: x_{4}: x_{5}: x_{6}\right) \rightarrow\left(x_{3}: x_{4}\right),
\end{aligned}
$$

respectively the rational map $F_{v}: \Sigma \rightarrow \mathbb{P}^{1}$, induced by the linear projection

$$
\left(x_{1}: x_{2}: x_{3}: x_{4}: x_{5}: x_{6}\right) \rightarrow\left(x_{5}: x_{6}\right) .
$$

We have that $\left(u_{0}(x): u_{1}(x)\right)=p_{u} \circ \pi=F_{u} \circ \phi_{1},\left(v_{0}(x): v_{1}(x)\right)=$ $p_{v} \circ \pi=F_{v} \circ \phi_{1}$. Moreover, $F_{u} \circ \phi_{1}$ is a morphism outside the finite set $R_{1} \cap R_{2}, F_{v} \circ \phi_{1}$ is a morphism outside $R_{3}$.

Hence $\pi=\left(F_{u} \circ \phi_{1}\right) \times\left(F_{v} \circ \phi_{1}\right): S \backslash R$ is a morphism of maximal rank, so $\phi_{1}$ is a local embedding outside of $R$.

2.3) Set for convenience $u:=p_{u} \circ \pi, v:=p_{v} \circ \pi$.

At the points of $R_{1} \backslash\left(R_{2} \cup R_{3}\right)$, then $y_{1}, u, v$ give local coordinates, so we are done; similarly for the points of $R_{2} \backslash\left(R_{1} \cup R_{3}\right)$.

At the points of $R_{3} \backslash\left(R_{1} \cup R_{2}\right)$, we need to show that $y_{3}$ and $u$ give local coordinates. Here, we make the remark that $D_{3}$ is a divisor of bidegree $(4,1)$, hence $p_{u}: D_{3} \rightarrow \mathbb{P}^{1}$ is an isomorphism. Hence $\delta_{3}, u$ give local coordinates at the points of $D_{3}$, and we infer that $y_{3}, u$, give local coordinates at the points of $R_{3} \backslash\left(R_{1} \cup R_{2}\right)$.

Claim 3: $\phi_{1}$ is injective. 
Assume that $\phi_{1}(x)=\phi_{1}\left(x^{\prime}\right)$. We observe preliminarily that this condition implies that, if $x \in R_{i}$, then also $x^{\prime} \in R_{i}$, since for instance $x \in R_{1} \Leftrightarrow x_{1}\left(\phi_{1}(x)\right)=x_{2}\left(\phi_{1}(x)\right)=0$.

3.1) Assume that $x, x^{\prime} \in S \backslash R$.

Then $\pi(x)=\pi\left(x^{\prime}\right)$, and since the elements of Galois group $G=$ $(\mathbb{Z} / 2)^{2}=\{ \pm 1\}^{3} /\{ \pm 1\}$ are determined by $\epsilon \in\{ \pm 1\}^{3}$, and each of them acts on $\phi_{1}(x)=\left(x_{1}: x_{2}: x_{3}: x_{4}: x_{5}: x_{6}\right)$ by

$$
\left(x_{1}: x_{2}: x_{3}: x_{4}: x_{5}: x_{6}\right) \mapsto\left(\epsilon_{1} x_{1}: \epsilon_{1} x_{2}: \epsilon_{2} x_{3}: \epsilon_{2} x_{4}: \epsilon_{3} x_{5}: \epsilon_{3} x_{6}\right),
$$

the action of $G$ on $\phi_{1}(x)$ has an orbit of cardinality 4 , which is precisely the cardinality of $\pi^{-1}(\pi(x))$.

Hence $\phi_{1}(x)=\phi_{1}\left(x^{\prime}\right) \Rightarrow x=x^{\prime}$.

3.2) Let $x, x^{\prime} \in R_{1} \backslash\left(R_{2} \cup R_{3}\right)$. Again $\pi(x)=\pi\left(x^{\prime}\right)$, and we see that the orbit of $\phi_{1}(x)$ under $G$ is the set of points

$$
\left(0: 0: \epsilon_{2} x_{3}: \epsilon_{2} x_{4}: \epsilon_{3} x_{5}: \epsilon_{3} x_{6}\right),
$$

which has cardinality 2 ; this is precisely the cardinality of $\pi^{-1}(\pi(x))$, so again we are done.

The case $x, x^{\prime} \in R_{2} \backslash\left(R_{1} \cup R_{3}\right)$ is completely analogous.

3.3) Let $x, x^{\prime} \in R_{3} \backslash\left(R_{1} \cup R_{2}\right)$. Again, the orbit of $\phi_{1}(x)$ under $G$ has cardinality 2 , so we are done if we show that $\pi(x)=\pi\left(x^{\prime}\right)$. On the set $R_{3}$, however, $\pi$ is not a morphism, so we argue differently.

We use instead that $u(x)=u\left(x^{\prime}\right)$, and that $p_{u}: D_{3} \rightarrow \mathbb{P}^{1}$ is an isomorphism to conclude that $\pi(x)=\pi\left(x^{\prime}\right)$.

3.4) If $x, x^{\prime} \in R_{3} \cap R_{2}$ (for $x, x^{\prime} \in R_{3} \cap R_{1}$ the argument is entirely similar), we proceed as follows.

We obtain again $u(x)=u\left(x^{\prime}\right)$, and since $p_{u}: D_{3} \rightarrow \mathbb{P}^{1}$ is an isomorphism, we get that $\pi(x)=\pi\left(x^{\prime}\right)$. However, the restriction of $\pi$ to $R_{3} \cap R_{2}$ is bijective, hence $x=x^{\prime}$.

3.5) Assume finally that $x, x^{\prime} \in R_{1} \cap R_{2}$ and use again that the restriction of $\pi$ to $R_{1} \cap R_{2} \pi$ is bijective. It suffices therefore to show that $\pi(x)=\pi\left(x^{\prime}\right)$.

The condition $\phi_{1}(x)=\phi_{1}\left(x^{\prime}\right)=\left(0: 0: 0: 0: y_{3} v_{0}: y_{3} v_{1}\right)$, since $y_{3}$ does not vanish on $R_{1} \cap R_{2}$, implies that $v(x)=v\left(x^{\prime}\right)$, so the points $\pi(x), \pi\left(x^{\prime}\right) \in D_{1} \cap D_{2}$ have the same $v$ coordinate.

Hence, by our assumption, $\pi(x)=\pi\left(x^{\prime}\right)$, exactly as desired.

Proposition 2.2. The hypotheses of theorem 2.1 define a non empty family of dimension $11+11+9=31$.

Proof. $D_{1}, D_{2}$ vary in a linear system of dimension $3 \times 4-1=11$, $D_{3}$ varies in a linear system of dimension $5 \times 2-1=9$.

The condition that the divisors intersect transversally is a consequence of the fact that each of the three linear systems embeds $Q$ in a projective space. 
The final condition amounts to the following: write

$$
\delta_{j}=u_{0}^{2} A_{j}(v)+u_{0} u_{1} B_{j}(v)+u_{1}^{2} C_{j}(v)=0, j=1,2 .
$$

We can view the coefficients of $\delta_{1}, \delta_{2}$, six degree three homogeneous polynomials in $v=\left(v_{0}: v_{1}\right)$, as giving a map $\psi$ of $\mathbb{P}^{1}$ inside the $\mathbb{P}^{5}$ with coordinates

$$
\left(A_{1}: B_{1}: C_{1}: A_{2}: B_{2}: C_{2}\right)
$$

parametrizing pairs of homogeneous polynomials of degree 2 in $u=$ $\left(u_{0}: u_{1}\right)$,

$$
\mathcal{P}_{j}=u_{0}^{2} A_{j}+u_{0} u_{1} B_{j}+u_{1}^{2} C_{j}=0, j=1,2 .
$$

Let $\Delta$ be the resultant

$\Delta:=\operatorname{Res}_{u}\left(\mathcal{P}_{1}, \mathcal{P}_{2}\right)=\left(A_{1} C_{2}-A_{2} C_{1}\right)^{2}+\left(B_{2} C_{1}-C_{2} B_{1}\right)\left(A_{1} B_{2}-A_{2} B_{1}\right)$.

The resultant $\Delta$ defines a hypersurface of degree 4 in $\mathbb{P}^{5}$, which is reduced and irreducible, being a non degenerate quadric in the variables $B_{1}, B_{2}$.

We can therefore choose the six polynomials in a general way so that the twisted cubic curve $\psi\left(\mathbb{P}^{1}\right)$ intersects $\Delta$ transversally in 12 distinct points.

We conclude because the image of $D_{1} \cap D_{2}$ via the projection $p_{v}$ is given by the 12 zeros of

$f\left(v_{0}: v_{1}\right):=\operatorname{Res}_{u}\left(\delta_{1}, \delta_{2}\right)=\Delta\left(A_{1}(v): B_{1}(v): C_{1}(v): A_{2}(v): B_{2}(v): C_{2}(v)\right)$,

and by our general choice these are 12 distinct points.

Remark 2.3. i) Taking into account the group of automorphisms of $Q=\mathbb{P}^{1} \times \mathbb{P}^{1}$, we see that the above family gives a locally closed subset of dimension 25 inside the moduli space of surfaces of general type. This dimension is more than the expected dimension $10 \chi(S)-2 K_{S}^{2}=$ $70-48=22$.

ii) since $H^{0}\left(\mathcal{O}_{Q}\left(D_{i}-L_{i}\right)=0\right.$ for each $i=1,2,3$, there are no natural deformations (Cat84, Cat99); it is not clear that our family yields an irreducible component of the moduli space, since the elementary method of cor. 2.20 and theorem 3.8 of [Cat84 do not apply.

\section{The CANONicAl Ring AS A MOdUle OVER THE SyMmetriC ALGEBRA $\mathcal{A}:=\operatorname{Sym}\left(\mathcal{R}_{1}\right)$}

Let us first of all look at the homomorphism $m_{2}: \operatorname{Sym}^{2}\left(\mathcal{R}_{1}\right) \rightarrow \mathcal{R}_{2}$, keeping track of the eigenspace decompositions, and using the notation $H^{0}\left(\mathcal{O}_{Q}(a, b)\right)=: V(a, b)$. We have then

$$
\begin{gathered}
\mathcal{R}_{1}=0 \oplus y_{1} V(1,0) \oplus y_{2} V(1,0) \oplus y_{3} V(0,1), \\
\mathcal{R}_{2}=V(4,3) \oplus y_{2} y_{3} V(1,1) \oplus y_{1} y_{3} V(1,1) \oplus y_{1} y_{2} V(2,0) .
\end{gathered}
$$


Since $V(1,0) \otimes V(0,1) \cong V(1,1)$, and we have a surjection $V(1,0) \otimes$ $V(1,0) \rightarrow V(2,0)$, the non trivial character spaces of $\mathcal{R}_{2}$ are in the image of $m_{2}: \operatorname{Sym}^{2}\left(\mathcal{R}_{1}\right) \rightarrow \mathcal{R}_{2}$.

Moreover, the kernel of $y_{1} V(1,0) \otimes y_{2} V(1,0) \rightarrow y_{1} y_{2} V(2,0)$ is 1dimensional, and provides a quadric $\{q(x)=0\}$ containing the canonical image $\Sigma$ of $S$. To simplify our notation, we directly assume that $S \subset \mathbb{P}^{5}$, via the canonical embedding.

Then the quadric that we obtain is: $q(x):=x_{2} x_{3}-x_{1} x_{4}$.

The trivial character space of $\mathcal{R}_{2}$ is isomorphic to $V(4,3)$ and contains the image of the subspace

$$
W:=y_{1}^{2} V(2,0) \oplus y_{2}^{2} V(2,0) \oplus y_{3}^{2} V(0,2) \subset \operatorname{Sym}^{2}\left(\mathcal{R}_{1}\right),
$$

which maps onto

$$
W^{\prime}:=\delta_{1} V(2,0)+\delta_{2} V(2,0)+\delta_{3} V(0,2) .
$$

Lemma 3.1. $W \rightarrow W^{\prime}$ is an isomorphism.

Proof. Assume that there is a kernel: then there are bihomogeneous polynomials $P_{1}, P_{2}, P_{3}$ such that $P_{1} \delta_{1}+\delta_{2} P_{2}=P_{3} \delta_{3}$.

Since $\delta_{3}$ does not vanish at the 12 points where $\delta_{1}=\delta_{2}=0, P_{3}=$ $P_{3}(v)$ should vanish on the projections of these 12 points under $p_{v}$. But this is a contradiction, since $P_{3}$ has degree 2 , while the 12 projected points are distinct. Hence $P_{3} \equiv 0$, and $\delta_{1} \mid P_{2}$, a contradiction again since $P_{2}$ has bidegree $(2,0)$.

Corollary 3.1. $S$ is contained in a unique quadric $\{q(x)=0\}$, and $m_{2}$ : $\operatorname{Sym}^{2}\left(\mathcal{R}_{1}\right) \rightarrow \mathcal{R}_{2}$ has image of dimension $21-1=20$ and codimension 11 .

Proof. $\quad \mathcal{R}_{m}$ has dimension $\operatorname{dim}\left(\mathcal{R}_{m}\right)=\chi(S)+\frac{1}{2} m(m-1) K_{S}^{2}=$ $7+12 m(m-1)$, which, for $m=2$, is equal to 31 .

We shall now choose $z_{1}, \ldots, z_{11} \in \mathcal{R}_{2}$ which, together with $\operatorname{Im}\left(m_{2}\right)$, generate $\mathcal{R}_{2}$. The elements $z_{1}, \ldots, z_{11}$ induce a basis of the quotient $\mathcal{R}_{2} / \operatorname{Im}\left(m_{2}\right)$.

Theorem 3.2. Let $\mathcal{A}:=\operatorname{Sym}\left(\mathcal{R}_{1}\right)$ be the coordinate ring of $\mathbb{P}^{5}$, and consider $\mathcal{R}$ as an $\mathcal{A}$-module. Then $1, z_{1}, \ldots, z_{11}$ is a minimal graded system of generators of $\mathcal{R}$ as an $\mathcal{A}$-module.

Proof. In view of the previous observations, it suffices to show that these elements generate $\mathcal{R}$.

Observe preliminarily that $V(a, b) \otimes V(c, d) \rightarrow V(a+c, b+d)$ is always surjective as soon as $a, b, c, d \geq 0$.

For $\mathcal{R}_{3}$, let us write:

$$
\mathcal{R}_{3}=y_{1} y_{2} y_{3} V(2,1) \oplus y_{1} V(5,3) \oplus y_{2} V(5,3) \oplus y_{3} V(4,4)
$$


The last three eigenspaces are in the image of $V(4,3) \otimes \mathcal{R}_{1} \subset \mathcal{R}_{2} \otimes$ $\mathcal{R}_{1} \rightarrow \mathcal{R}_{3}$.

Also the first summand is in the image of $y_{1} V(1,0) \otimes y_{2} y_{3} V(1,1)$.

The same argument works for $\mathcal{R}_{2 m+1}$, while for $\mathcal{R}_{2 m+2}$ we find surjections

$$
H^{0}\left(\mathcal{O}_{Q}(N)\right) \otimes w_{i} H^{0}\left(\mathcal{O}_{Q}\left(m N-L_{i}\right)\right) \rightarrow w_{i} H^{0}\left(\mathcal{O}_{Q}\left((m+1) N-L_{i}\right)\right),
$$

and

$$
H^{0}\left(\mathcal{O}_{Q}(N)\right) \otimes H^{0}\left(\mathcal{O}_{Q}(m N)\right) \rightarrow H^{0}\left(\mathcal{O}_{Q}((m+1) N)\right) .
$$

Hence the claimed result follows by induction on $m$.

Remark 3.3. 1) Since $\operatorname{dim} \mathcal{R}_{3}=79, \operatorname{dim} \mathcal{A}_{3}=56$, we see that the 12 minimal generators of the module admit $56+6 \times 11-79=43$ relations in degree 3.

The module $\mathcal{R}$ is Cohen-Macaulay, hence it has a length 3 minimal graded resolution.

2) In general, $\operatorname{dim} \mathcal{R}_{m}=7+12 m(m-1)$,

$$
\operatorname{dim} \mathcal{A}_{m}=\left(\begin{array}{c}
m+5 \\
m
\end{array}\right)
$$

hence the image of $\mathcal{A}_{m} \rightarrow \mathcal{R}_{m}$ has dimension less than or equal (because $S$ is contained in a quadric) to $\operatorname{dim} \mathcal{A}_{m}-\operatorname{dim} \mathcal{A}_{m-2}$.

Hence the Hartshorne-Rao module $\oplus_{m} H^{1}\left(\mathcal{I}_{S}(m)\right.$ is non zero in all degrees $m=2,3,4,5,6$. This shows that the bundle $\mathcal{E}$ contructed by Walter using the Horrocks correspondence should be rather interesting.

\section{REFERENCES}

[BC08] I. Bauer, F. Catanese, A volume maximizing canonical surface in 3-space. Comment. Math. Helv. 83, No. 1, 387-406 (2008).

[Cat79] F. Catanese, On Severi's proof of the double point formula. Commun. Algebra 7, (1979) 763-773.

[Cat84] F. Catanese, On the Moduli Spaces of Surfaces of General Type, J. Differential Geom. 19 (1984), 483-515.

[Cat97] F. Catanese, Homological algebra and algebraic surfaces, AMS Proc. Symp. Pure Math 62.1(1997), 3-56.

[Cat99] F. Catanese, Singular bidouble covers and the construction of interesting algebraic surfaces. AMS Cont. Math. 241 (1999), 97 - 120.

[Cat00] F. Catanese, Fibred surfaces, varieties isogenous to a product and related moduli spaces, Amer. J. Math. 122 (2000), no. 1, 1-44.

[Hart77] R. Hartshorne, Algebraic geometry, Springer GTM 52, (1977).

[Hor81] E. Horikawa, Algebraic surfaces of general type with small $c_{1}^{2}, V$, J. Fac. Sci. Univ. Tokyo, Sect. A. Math 283 (1981), 745-755.

[Kap-Kap15] G. Kapustka, M. Kapustka Bilinkage in codimension 3 and canonical surfaces of degree 18 in $\mathbb{P}^{5}$, arXiv: 1312.2824, to appear in the Ann. Sc. Norm. Super. Pisa, (2015).

[Kon93] K. Konno, Non-hyperelliptic fibrations of small genus and certain irregular canonical surfaces, Ann. Sc. Norm. Super. Pisa, Cl. Sci. (IV) 20 (4), (1993), 575-595. 
[Sev01] F. Severi, Intorno ai punti doppi impropri di una superficie generale dello spazio a quattro dimensioni e ai suoi punti tripli apparenti Rend. Circ. Mat. Palermo, 15 (1901), 33-51.

[Wal96] C. Walter, Pfaffian subschemes. J. Alg. Geom., 5 (1996), 671-704.

[Xiao87] G. Xiao, Fibered algebraic surfaces with low slope, Math. Ann. 276 (1987), 449-466.

Lehrstuhl Mathematik VIII, Mathematisches Institut Der UniverSITÄт BAYREUth, NW II, Universitätssstr. 30, 95447 BAYREUth

E-mail address: fabrizio.catanese@uni-bayreuth.de 